\title{
ABUNDÂNCIA RELATIVA E DISTRIBUIÇÃO ESPAÇO-TEMPORAL DE MICROPOGONIAS FURNIERI (DESMAREST) E CYNOSCION LEIARCHUS (CUVIER) (PERCIFORMES, SCIAENIDAE) NO MANGUEZAL DO ITACORUBI, SANTA CATARINA, BRASIL ${ }^{1}$
}

\author{
Mauricio Hostim-Silva ${ }^{2,3}$ \\ Gisela Costa Ribeiro ${ }^{2}$ \\ Leandro Clezar ${ }^{2}$ \\ Blanca Sierra de Ledo ${ }^{2}$
}

\begin{abstract}
RELATIVE ABUNDANCE AND SPATIAL DISTRIBUTION OF MICROPOGONIAS FURNIERI (DESMAREST) E CYNOSCION LEIARCHUS (CUVIER) (PERCIFORMES, SCIAENIDAE) IN THE MANGROVE OF ITACORUBI, SANTA CATARINA, BRASIL. Attempting to contribute with the knowledge about the bioecological aspects concerning the youth forms of M. furnieri (Desmarest, 1823) and C. leiarchus (Cuvier, 1830) from Itacorubi mangrove, 15 samplings were taken from January 1988 to March 1989. Using casting and entangle nets, a total of 308 individuals from these species were captured. Their abundance were: $M$. furnieri $N=197(64,0 \%)$; C. leiarchus $\mathrm{N}=111(36,0 \%)$. The relative frequency and range of total weights and lengths were studied. Concerning spatial distribution of the species, it was observed that M. furnieri occurred in all areas and C. leiarchus was not captured only in "D" area (Sertão River). During the anual cycle the two species occurred jointly. The results emphasize other authors statements that consider this mangrove as a "natural growing enviroment" which is highly important for the region.

KEY WORDS. Sciaenidae, mangrove, abundance, distribution
\end{abstract}

A família Sciaenidae, com ampla distribuição geográfica e grande número de espécies, integra a ictiofauna dos cinco continentes, representando uma das famílias mais expressivas da atualidade (CHAO, 1978; SOUZA \& SANTOS, 1988).

Embora registrando a sua ocorrência para águas mais profundas, a maioria das espécies habita águas costeiras e rasas, próximas de estuários, baías, desembocaduras de grandes rios e até mesmo em áreas de recifes de coral, em fundos de areia ou lama, constituindo um dos mais importantes recursos

1) Realizado a partir dos convênios CIRM/Universidade Federal de Santa Catarina e Núcleo de Estudos do Mar.

2) Universidade Federal de Santa Catarina, Núcleo de Estudos do Mar, Caixa Postal 476, 88049-970 Florianópolis, Santa Catarina, Brasil.

3) Universidade Vale do Itajaí, Facudade de Filosofia Ciências e Letras, Caixa Postal 360, 88300-000 Itajaí, Santa Catarina, Brasil. 
pesqueiros demersais costeiros do Atlântico e de grande relevância para o homem como fonte alimentar (CHAO \& MUSICK, 1977; MENEZES \& FIGUEIREDO, 1980). Pela condição de ser bastante abundante e ter uma relativa importância pesqueira, GIANNINI (1989) cita um bom número de trabalhos realizados sobre Sciaenidae para a costa Sul e Sudeste do Brasil, possibilitando com isso um maior conhecimento do ciclo de vida, biologia e ecologia.

Embora os ecossistemas de manguezais constituam ambientes conspícuos, considerados criadouros naturais da maior importância, poucos estudos têm sido executados no que diz respeito às comunidades ícticas (AVELINE,1980).

Desta maneira o presente trabalho tem como objetivo verificar a coocorrência das espécies M. furnieri (Desmarest, 1823) e C. leiarchus (Cuvier, 1830) no Manguezal do Itacorubi, através da análise da variação sazonal e espacial da abundância, fornecendo subsídios para a preservação deste ambiente e das espécies que nele habitam.

\section{MATERIAL E MÉTODOS}

O presente trabalho foi realizado no Manguezal do Itacorubi (27 34 '14'

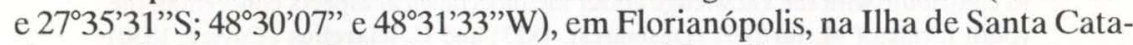
rina, onde foram estabelecidas quatro áreas, identificadas como: $\mathrm{A}, \mathrm{B}, \mathrm{C}, \mathrm{e} \mathrm{D}$ (Fig. 1). As coletas foram realizadas mensalmente, de janeiro de 1988 a março de 1989, em período noturno, com duração média de seis horas. Os peixes foram capturados em todas as áreas utilizando-se tarrafas com 50 e $20 \mathrm{~mm}$ de malha (nós opostos) e com esforço de pesca de 5 e 10 lances por área, respectivamente. $\mathrm{Na}$ área "B" (confluência dos Rios Itacorubi e Sertão), a coleta foi incrementada com o uso de mais um artefato de pesca, uma rede de espera (tipo feiticeira) com três malhas, sendo a central com $13 \mathrm{~mm}$ e as laterais com $60 \mathrm{~mm}$, ficando esta rede, em média, seis horas de permanência.

Em laboratório os exemplares foram triados, identificados com um número e registrado o comprimento total com ictiômetro (prec. $0,50 \mathrm{~cm}$ ) e peso total foi obtido com balança eletrônica (prec. $0,01 \mathrm{~g}$ ) de cada indivíduo a fresco. $\mathrm{Na}$ análise da freqüência $(\mathrm{Fa})$ e constância de ocorrência $(\mathrm{C})$ das espécies foram considerados a totalidade dos indivíduos coletados durante o período de amostragens, utilizando-se para isto os índices de Freqüência de Glemarec (1964) e de Constância segundo Dajoz (1978). No que se refere a variação temporal, abundância e freqüência, além dos meses amostrados, se utilizou o agrupamento arbitrário por estação do ano. Desta forma o verão corresponde aos meses de: dezembro, janeiro e fevereiro; outono: março, abril e maio; inverno: junho, julho e agosto; primavera: setembro, outubro e novembro. Os valores de comprimento total foram agrupados em classes de $10 \mathrm{~mm}$ e estimados as suas freqüências percentuais no ciclo anual. Foi calculado a amplitude de comprimento total, as modas, a amplitude de peso total, peso total médio e biomassa total por área e por estação do ano. Para a análise da co-ocorrência das espécies, utilizou-se das frequiências relativas em cada área e durante o ciclo anual. 


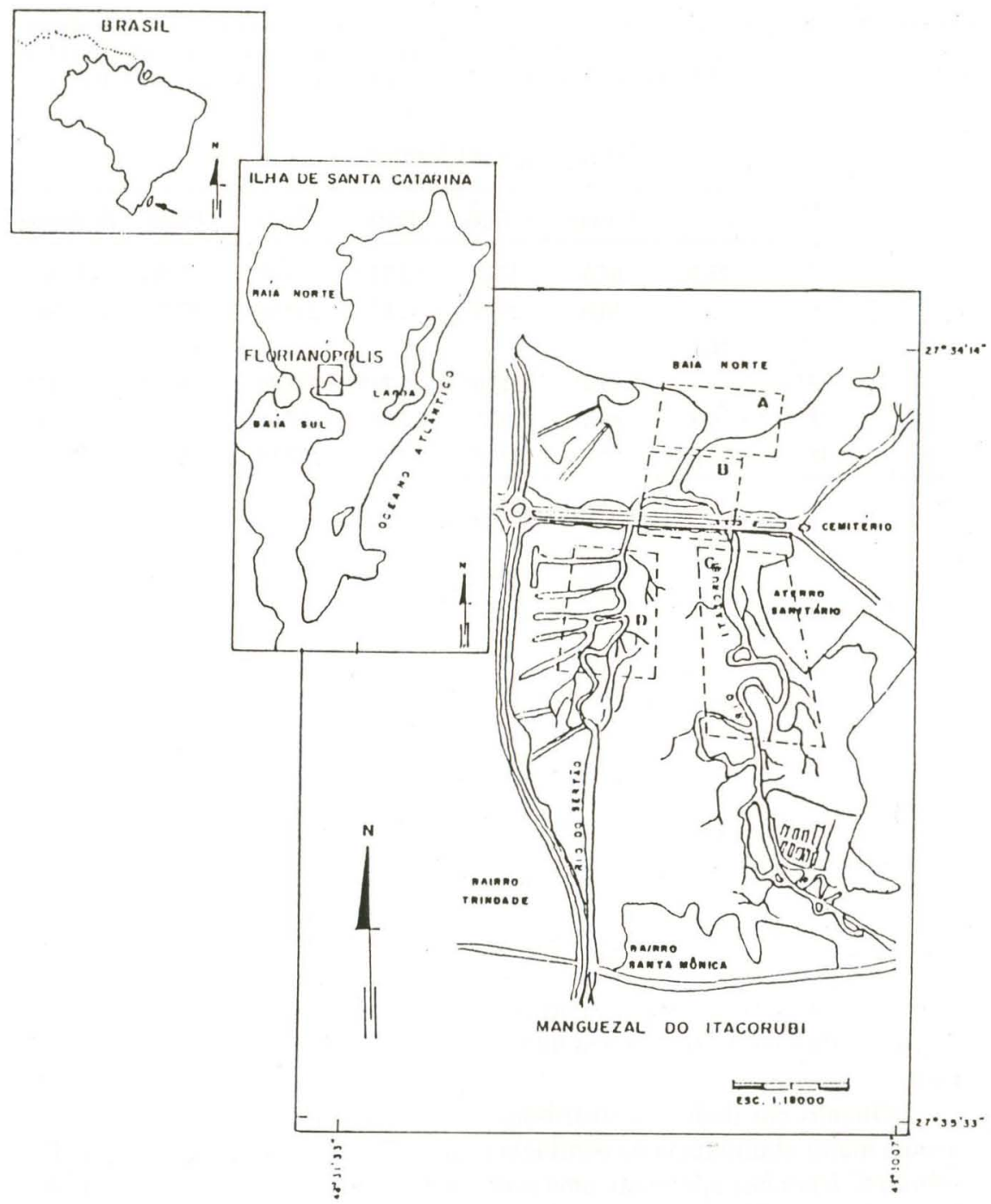

Fig. 1. Manguezal do Itacorubi, Ilha de Santa Catarina, SC, Brasil. Localização das áreas de coletas.

\section{RESULTADOS E DISCUSSÃO}

Com relação à abundância das espécies, dos 308 indivíduos coletados (Tabs I e II), M. furnieri contribuiu com 197 exemplares e C. leiarchus com 111, sendo que ambas foram consideradas constantes $(\mathrm{C}>50 \%)$. No entanto, HOSTIM-SILVA (observação pessoal) realizando um projeto piloto para o estudo de Sciaenidae neste manguezal no período de 1986 a 1987, constatou que $M$. furmieri foi a espécie mais abundante e constante $(83,3 \%)$, sendo que $C$. 
Tabela I. Abundância numérica (N) e relativa (\%); comprimento total máximo (CTmáx.) e mínimo (CTmín.) em milímetros; peso total máximo (Pmáx.), mínimo (Pmín.), médio (Pméd) e biomassa em gramas, obtidos para M. furnieri e C. leiarchus por área de coleta no Manguezal do Itacorubi, SC.

\section{Micropogonias furnieri}

\begin{tabular}{ccccccccc}
\hline Área & N & $\%$ & CTmín & CTmáx & Pmín & Pmáx & Pméd & Biomassa \\
\hline A & 37 & 25,3 & 67,0 & 176,0 & 2,75 & 61,90 & 12,99 & 480,85 \\
& 82 & & 66,0 & 293,0 & 2,67 & 259,84 & 37,72 & 2682,94 \\
B & & 56,2 & & & & & & \\
& $51^{*}$ & & $95,0^{*}$ & $313,0^{*}$ & $6,70^{*}$ & $335,90^{*}$ & $128,00^{*}$ & $6528,41^{*}$ \\
C & 9 & 6,3 & 49,0 & 193,0 & 1,04 & 69,66 & 13,75 & 123,74 \\
D & 18 & 12,2 & 98,0 & 174,0 & 8,60 & 52,14 & 18,71 & 336,75 \\
\hline
\end{tabular}

Cynoscion leiarchus

\begin{tabular}{ccccccccc}
\hline Área & N & $\%$ & CTmín & CTmáx & Pmín & Pmáx & Pméd & Biomassa \\
\hline A & 1 & 3,4 & - & 114,0 & - & 16,10 & - & 16,10 \\
& 8 & & 110,0 & 170,0 & 14,96 & 54,50 & 30,72 & 245,73 \\
B & & 27,6 & & & & & & \\
& $82^{*}$ & & $109,0^{*}$ & $258,0^{*}$ & $16,61^{*}$ & $181,61^{*}$ & $57,25^{*}$ & $4694,70^{*}$ \\
C & 20 & 69,0 & 67,0 & 206,0 & 2,31 & 108,61 & 42,50 & 850,04 \\
D & - & - & - & - & - & - & - & - \\
\hline
\end{tabular}

* Dados de rede-de-espera.

leiarchus, por sua vez, foi menos abundante e considerada espécie acessória (33,3\%). Talvez esta discrepância seja conseqüência das capturas em 1986/1987 terem sido em período diurno, enquanto as do presente estudo foram sempre noturnas e de maior abundância numérica, utilizando os mesmos artefatos de pesca.

Quanto aos dados de distribuição espacial, verificou-se que $M$. furnieri teve sua maior abundância na confluência dos rios Sertão e Itacorubi (área "B"). Embora C. leiarchus apresente uma certa preferência pela área ao longo do Rio Itacorubi (área "C"), quando analisa-se a abundância obtida com a rede de espera, a área de confluência passa a ser a mais representativa (Tab. I). Entretanto, não parece haver exclusividade para as espécies, pois verifica-se a presença de $M$. furnieri em todas as áreas e C. leiarchus ausente somente ao longo do Rio Sertão (Tab. I). SILVA (1982), encontrou M. furnieri distribuído por toda a região estuarina de Tramandaí (Rio Grande do Sul), porém não indicando as áreas de maior abundância. Este mesmo autor e CHAO et al. (1982) não registraram a ocorrência de $C$. leiarchus tanto para a região estuarina de Tramandaí como para a Lagoa dos Patos (Rio Grande do Sul), respectivamente. 
Tabela II. Abundância numérica (N) e relativa (\%); comprimento total máximo (CTmáx.) e mínimo (CTmín.) em milímetros; peso total máximo (Pmáx.), mínimo (Pmín.), médio (Pméd) e biomassa em gramas, obtidos para M. furnieri e C. leiarchus por estação do ano no Manguezal do Itacorubi, SC.

Micropogonias furnieri

\begin{tabular}{cccclcccc}
\hline Estação & $\mathrm{N}$ & $\%$ & CTmín & CTmáx & Pmín & Pmáx & Pméd & Biomassa \\
\hline \multirow{2}{*}{ VER } & 49 & 33,6 & 49,0 & 193,0 & 1,04 & 69,66 & 17,30 & 847,53 \\
& $28^{*}$ & $54,9^{*}$ & $129,0^{*}$ & $313,0^{*}$ & $22,00^{*}$ & $335,90^{*}$ & $140,37^{*}$ & $3930,47^{*}$ \\
\multirow{2}{*}{ OUT } & 77 & 52,7 & 67,0 & 293,0 & 2,75 & 259,84 & 28,64 & 2229,50 \\
& $10^{*}$ & $19,6^{*}$ & $95,0^{*}$ & $275,0^{*}$ & $6,70^{*}$ & $226,33^{*}$ & $109,55^{*}$ & $1095,51^{*}$ \\
INV & 2 & 1,4 & 68,0 & 120,0 & 2,79 & 19,74 & 11,27 & 22,53 \\
& $5^{*}$ & $9,8^{*}$ & $118,0^{*}$ & $275,0^{*}$ & $16,90^{*}$ & $233,83^{*}$ & $150,81^{*}$ & $754,07^{*}$ \\
PRI & 18 & 12,3 & 68,0 & 185,0 & 3,10 & 59,52 & 29,15 & 524,72 \\
& $8^{*}$ & $15,7^{*}$ & $154,0^{*}$ & $252,0^{*}$ & $35,80^{*}$ & $165,81^{*}$ & $93,54^{*}$ & $748,36^{*}$ \\
\hline
\end{tabular}

Cynoscion leiarchus

\begin{tabular}{ccccccccc}
\hline Estação & N & $\%$ & CTmín & CTmáx & Pmín & Pmáx & Pméd & Biomassa \\
\hline \multirow{2}{*}{ VER } & 18 & 62,1 & 67,0 & 185,0 & 2,31 & 75,23 & 28,64 & 515,59 \\
& $56^{*}$ & $68,3^{*}$ & $121,0^{*}$ & $258,0^{*}$ & $19,86^{*}$ & $181,61^{*}$ & $49,76^{*}$ & $2786,40^{*}$ \\
\multirow{2}{*}{ OUT } & 11 & 37,9 & 114,0 & 206,0 & 14,96 & 108,01 & 54,21 & 596,28 \\
& $23^{*}$ & $28,1^{*}$ & $109,0^{*}$ & $215,0^{*}$ & $16,61^{*}$ & $124,79^{*}$ & $68,62^{*}$ & $1578,25^{*}$ \\
INV & - & - & - & - & - & - & - & - \\
& $2^{*}$ & $2,4^{*}$ & $195,0^{*}$ & $215,0^{*}$ & $78,25^{*}$ & $121,23^{*}$ & $99,74^{*}$ & $199,48^{*}$ \\
PRI & - & - & - & - & - & - & - & - \\
& $1^{*}$ & $1,2^{*}$ & - & $231,0^{*}$ & - & $130,57^{*}$ & - & $130,57^{*}$ \\
\hline
\end{tabular}

* Dados de rede-de-espera.

Na Baía de Santos (São Paulo), M. furnieri mostrou uma tendência a maiores capturas associadas a zonas mais rasas das áreas internas, embora tenha apresentado também grandes capturas nas zonas mais profundas (GIANNINI \& PAIVA FILHO, 1990). No entanto, BARBIERI (1986), destaca que a distribuição de juvenis desta espécie é muito ampla quando se trata de ambiente estuarino. Segundo Érico Porto Filho (comunicação pessoal), na área de confluência (área "B"), onde obtivemos maior abundância há consideráveis porcentagens de areia fina; na área do Rio Sertão (área "D"), onde só ocorreu $M$. furnieri, o silte aparece em grandes proporções (Tab. I). RIBEIRO NETO \& PAIVA FILHO (1989) afirmam que nas áreas com sedimentos variando de silte fino e areia muito fina, a abundância e o número de espécies são màiores que nas áreas com sedimento variando de areia muito fina e areia fina, não sendo coincidentes com nossos resultados. No entanto também temos que levar em consideração o stress ambiental que o Manguezal do Itacorubi vem sofrendo, 
principalmente pelas modificações nos seus canais.

Os resultados obtidos em relação ao ciclo sazonal demonstraram que $M$. furnieri e $C$. leiarchus co-ocorreram durante todo o ano (Tab. II). PAIVA-FILHO et al. (1987), no complexo Baía-Estuário de Santos e São Vicente (São Paulo), verificaram que $M$. furnieri apareceu em todas as estações do ano, enquanto $C$. leiarchus só não apareceu na primavera. Na Lagoa dos Patos e região costeira adjacente, $M$. furnieri também foi registrada para todo ciclo anual (CHAO et al., 1982). VAZZOLER (1963) no estudo de deslocamentos sazonais da "corvina" relacionados com as massas de água, afirma que durante o verão as maiores concentrações de $M$. furnieri acham-se numa latitude $31^{\circ} \mathrm{S}$ para o sul, sendo que durante o inverno esta espécie tem suas maiores concentrações nas latitudes delimitadas por $27^{\circ} \mathrm{S}$ e $31^{\circ} \mathrm{S}$. A localização geográfica do manguezal estudado, próximo aos $27^{\circ} \mathrm{S}$, coloca este sistema dentro da área com maiores abundâncias para a espécie durante o inverno, o que não é coincidente com nossos resultados, que demonstraram ser o outono a época de maior abundância.

A distribuição em classes de comprimento total (Fig. 2), apresentou amplitudes de $40-310 \mathrm{~mm}$ e as maiores freqüências em 80 e $150 \mathrm{~mm}$ para $M$. furnieri; C. leiarchus amplitudes de $60-250 \mathrm{~mm}$, com maior freqüência em $170 \mathrm{~mm}$. Não foi verificado um padrão de distribuição por classe de comprimento no Manguezal do Itacorubi ou mesmo durante o ciclo sazonal para as espécies estudadas (Tabs I e II). Porém, GIANNINI \& PAIVA FILHO (1990), estudando a Baía de Santos, verificaram que jovens de $M$. furnieri foram capturados com maior eficiência pelos arrastos de praia a partir da primavera no lado leste do setor de praias (águas mais quentes e de salinidade mediana). Com o aumento do tamanho, os peixes passaram a ocupar as regiões mais afastadas das margens, sendo capturadas pelos arrastos de fundo junto aos setores mais internos, principalmente no verão. BARBIERI (1986) sugeriu o mesmo padrão de migração para a "corvina" juvenil da Lagoa dos Patos.

A rede de espera captura indivíduos quando estes vão de encontro à suas malhas e ficam presos pelo corpo, determinando um decréscimo da probabilidade de captura nos extremos da distribuição de comprimento do estoque, e um aumento desta a medida que o tamanho do indivíduo tende para um valor central (FONTENELES-FILHO, 1989). Segundo as observações de LAMOTTE \& BORLIERI (1971), ao utilizar-se sempre a mesma rede em vários locais, as variações foram consideráveis e podem corresponder a mudanças na composição ou na estrutura da fração de população amostrada. Provavelmente, a ausência de um padrão de distribuição espaço-temporal nas classes de comprimento seja conseqüência principalmente da seletividade dos artefatos de pesca utilizados, especialmente pelo emprego da rede de espera em uma única área, ou mesmo, pela dificuldade de utilização de rede de arrasto em áreas de manguezal, como foi empregado por GIANNINI \& PAIVA-FILHO (1990) e BARBIERI (1986) em suas amostragens.

Quanto à biomassa (Tabs I e II), para $M$. furnieri encontrou-se o menor de peso igual a $1,04 \mathrm{~g}$ e o maior $335,90 \mathrm{~g}$ e C. leiarchus apresenta o menor peso 


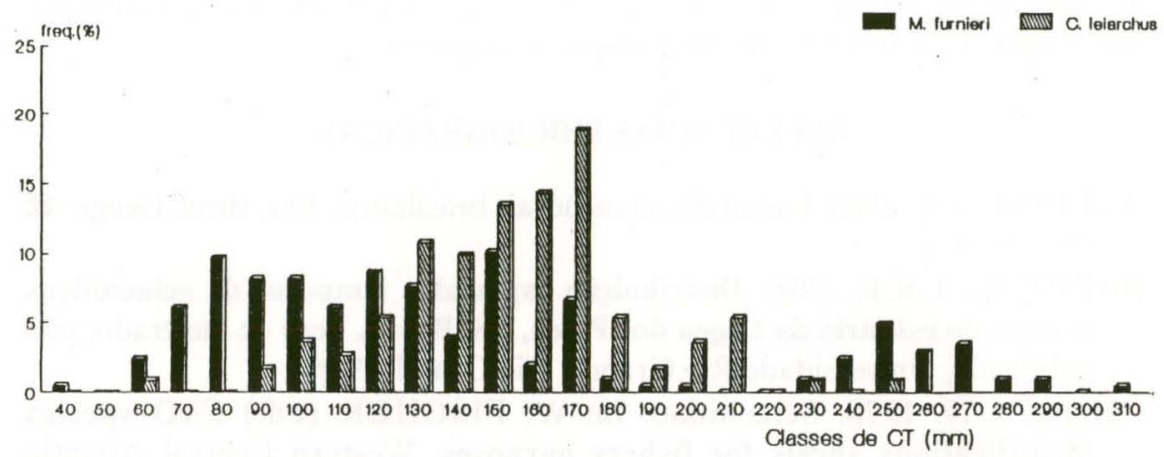

Fig. 2. Freqüência relativa (\%) de indivíduos de $\mathbf{M}$. furnieri e C. leiarchus por classe de comprimento total $(\mathrm{mm})$ para o período de janeiro de 1988 a março de 1989.

igual 2,31g e o maior 181,.61g. Para ambas as espécies seus extremos foram registrados no verão, sendo os menores valores para a área do Rio Itacorubi (área " $C^{\prime \prime}$ ) e os maiores na área de confluência (área "B"). Essas observações diferem um pouco das registradas por HOSTIM-SILVA (observação pessoal), pois o exemplar de menor peso $(4,50 \mathrm{~g})$ e o de maior peso $(209,4 \mathrm{~g})$ de $M$. furnieri foram capturados no Rio Itacorubi e no outono. C. leiarchus por sua vez, obteve o menor peso $(13,1 \mathrm{~g})$ para as áreas de desembocadura junto à Baía Norte e área de confluência dos rios, ambos no verão, enquanto o de maior peso $(56,6 \mathrm{~g})$, coletado na área de confluência dos rios e também no verão. Os resultados de abundância de $C$. leiarchus são coincidentes com os registrados por CLEZAR et al. (1991) para Cetengraulis edentulus (Cuvier, 1828) (Engraulididae), onde estas "manjubas" apresentaram maiores abundâncias nas áreas de confluência e ao longo do Rio Itacorubi, bem como para as estações de verão e outono. No sistema costeiro do litoral de Ubatuba (São Paulo), SOARES et al. (1989) identificaram três grupos de Sciaenidae com características tróficas distintas, sendo que as espécies do gênero Cynoscion (Gill, 1861) estão em dois grupos considerados comedores de peixes. É possível que exemplares jovens desta espécie entrem no Manguezal nestas épocas do ano e alimentem-se principalmente de "engraulídeos". Para a comprovação de tal fato, será necessário além de um estudo da ecologia trófica de $C$. leiarchus, uma análise da co-ocorrência espaço-temporal das espécies. Estudos semelhantes também deverão ser realizados para a espécie $M$. furnieri, visto que esta demonstrou uma ampla ocupação das áreas dentro do manguezal e durante todo o ciclo anual.

A grande abundância de indivíduos jovens de ambas as espécies no Manguezal do Itacorubi, vem enfatizar as atribuições sobre a sua função de criadouro natural e a importância de sua preservação. 
de campo. Às amigas Cláudia Machado e Gisele de Souza pela organização dos manuscritos e ao MsC. Jorge E. Kotas (CEPSUL/IBAMA) pelas valiosas sugestões.

\section{REFERÊNCIAS BIBLIOGRÁFICAS}

AVELINE, L.C. 1980. Fauna dos manguezais brasileiros. Rev. Bras. Geogr. 42 (4): 786-821.

BARBIERI, L.R.R. 1986. Distribuição espacial e temporal de sciaenídeos juvenis do estuário da Lagoa dos Patos, RS, Brasil. Tese de mestrado, não publicada, Universidade Rio Grande, Rio Grande, 98p.

CHAO, L.N. 1978. Sciaenidae. In: W. FISCHER (ed.) FAO species identifications sheets for fishery purposes. Western Central Atlantic (fishing area 31). Roma, FAO, vol. 4.

CHAO, L.N. \& J.A. MUSICK. 1977. Life history, feeding habitats and funcional morfology of juvenile sciaenid fishes in the York River estuary, Virginia. Fish. Bull. U.S. 75 (4): 657-702.

CHAO. L.N.; L.E. PEREIRA.; J.P. VIEIRA.; M.A. BENVENUTI \& L.P.R. CUNHA. 1982. Relação preliminar dos peixes estuarinos e marinhos da Lagoa dos Patos e região costeira adjacente, Rio Grande do Sul. Brasil. Atlântica. 5 (1): 67-75.

CLEZAR, L; G.C. RIBEIRO \& M. SILVA. 1991. Aspectos da biologia de Cetengraulis edentulus (Cuvier, 1828) (Osteichthyes: Clupeiformes) no Manguezal do Itacorubi, SC, Brasil. Res. IX Encon. Bras. Ictiologia, Maringá, p. 105.

DAJOZ, R. 1978. Ecologia Geral. Petrópolis, Vozes/Editora da USP. 474p.

FONTENELES FILHO, A.A. 1989. Recursos pesqueiros: Biologia e dinâmica populacional. Fortaleza, Impr. Oficial do Ceará, 296p.

GIANNINI, R. 1989. Distribuição temporal e espacial e aspectos bioecológicos da família Sciaenidae na Baía de Santos, SP, Brasil. Tese de mestrado, não publicada, Inst. Oceanográfico, Universidade de São Paulo, São Paulo, 203p.

GIANNINI, R. \& A.M. PAIVA FILHO. 1990. Os Sciaenidae (Teleostei: Perciformes) da Baía de Santos (SP), Brasil. Bolm. Inst. oceanogr., São Paulo, 38 (1): 69-86.

GLEMEREC, M. 1964. Bionomie benthique de la partie oriental du Morbihan. Cah. Biol. Mar. Roscoff. 5 (1): 33-96.

LAMOTTE, M. \& F. BOURLiERE. 1971. Problemes d'ecologie: l'echantillonage des peuplementes animaux des milieux aquatiques. Publication sous les auspeces du Comitê Français du Programme Biologique International, Masson \& Cie, 294p.

MENEZES, N.A. \& J.L. FIGUEIREDO. 1980. Manual de peixes marinhos do sudeste do Brasil. IV. Teleostei (3). São Paulo, Mus. Zool. Univ. São Paulo, 96p.

PAIVA-FILHO, M.A.; R. GIANNINI; F.B. RIBEIRA-NETO \& J.M.M. SCHMIEGELOW. 1987. Ictiofauna do complexo Baía-Estuário de Santos 
e São vicente, SP, Brasil. Relat. int. Inst. Oceanogr. Univ. São Paulo (17): $1-10$.

RIBEIRO NETO, F.B. \& A.M. PAIVA FILHO. 1989. Estrutura da comunidade de peixes da Baía de Santos, SP, Brasil. Res. I Simp. Oceanografia., Inst. Oceanogr. Univ. São Paulo, São Paulo, p. 132.

SILVA, C.P. 1982. Ocorrência, distribuição e abundância de peixes na região estuarina de Tramandaí, Rio Grande do Sul. Atlântica 5: 49-66.

SOARES, L.S.H.; C.L.D.B. ROSSI-WONGTSCHWSKI; M.J. REYNA; M.A. GASALLA; M.V. ARRASA \& M.A.T. RIOS. 1989. Ecologia trófica da Ictiofauna do sistema costeiro do litoral de Ubatuba, São Paulo, Brasil. I: Sciaenidae. Res. I Simp. Oceanografia, Inst. Oceanogr. Univ. São Paulo, São Paulo, p. 29.

SOUZA, I.C. \& R.S. SANTOS. 1988. Osteologia do complexo craniano de Pachypops adspersus (Steindachner) (Osteichthyes, Sciaenidae). Biotemas 1 (1): 33-56.

VAZZOLER, A.E.A. DE M. 1963. Deslocamentos sazonais da corvina relacionados com as massas d'água. Contrçôes. Inst. Oceanogr. Univ. São Paulo, Sér. Ocean. Biol. (5):1-8.

Recebido em 25.II.1993; aceito em 13.VIII.1993. 